\title{
Spinal Primary Neoplasia/Metastasis
}

Primary and metastatic neoplasia may involve the spine in patients in all age categories. Sometimes presenting with acute but potentially reversible neurological deficit, such patients may benefit from emergency MRI, used to visualize the spinal compartment to assess the extent and degree of spinal cord or cauda equina compression frequently associated with such disease (Daffner et al., 1986; Beltran et al., 1987; Colman et al., 1988; Avrahami et al., 1989; Carmody et al., 1989; Algra et al., 1991; Coletti et al. 1991).

\section{CONVENTIONAL AND FAST SPIN ECHO}

Although findings of destructive, mass-forming, and marrow-replacing neoplastic disease can often be used to diagnose advanced involvement of the spinal column, contrast agents are almost always utilized in this subset of patients to determine clearly the focus and extent of the enhancing, solid portions of epidural neoplasia as an indication of the degree and regional expression of the neural tissue compression prior to decompressive surgery and/or chemo-radiation therapy (Jinkins et al., 1998).

The following sequences comprise the preferred protocol for high-field MR machines. In all cases intravenous (i.v.) contrast-enhanced protocols are mandatory to evaluate the integrity of the blood-nerve and blood-cord barrier in certain cases of suspected central nervous system (CNS) infection, or in cases of epidural and/or spinal column/paraspinal infection (e.g., spondyloclodiscitis and perispinal abscess/phlegmon; Sze et al., 1988; Sugimura et al., 1991; Ragland et al., 1996).

Table A8.5.1 lists the hardware necessary to perform the procedure, along with appropriate parameters. The available gradient strength will depend on the scanner, and the echo times (i.e., $T_{\mathrm{E}}$ ) given in other tables will be varied accordingly (i.e., the smaller the gradient strength, the longer the echo time for a particular scan).

The entire protocol should take 45 to $50 \mathrm{~min}$ to complete.

NOTE: Be sure that technicians and nurses have immediate access to any emergency equipment that may be relevant to a given study, or that may be needed for a particular patient, such as crash carts or oxygen.

\section{Materials}

Normal saline $(0.9 \% \mathrm{NaCl})$, sterile

Extravascular contrast agent (e.g., Magnevist, Omniscan, or Prohance)

\section{Set up patient and equipment}

1. Interview (screen) the patient to ensure that he or she has no contraindications such as cardiac pacemakers or other implants containing ferromagnetic materials. Also be sure to find out if the patient has any health conditions that may require the presence

Table A8.5.1 Equipment Parameters for Spine Imaging in Cases of Suspected Primary or Metastatic Neoplasia

\begin{tabular}{|c|c|}
\hline Coil type & $\begin{array}{l}\text { Cervical, thoracic, lumbar: phase array } \\
\text { surface coil (or other depending upon } \\
\text { machine compatibility and availability) }\end{array}$ \\
\hline Gradient coil strength & $25 \mathrm{mT} / \mathrm{m}$ (or whatever the system permits) \\
\hline Flow compensation pulse & Optional (any level, if available) \\
\hline Peripheral gating & Optional (thoracic spine only) \\
\hline Respiratory gating & Optional (thoracic spine only) \\
\hline Use of contrast agents & Yes \\
\hline
\end{tabular}




\begin{tabular}{ll}
\hline Patient position & Supine \\
Scan type & Gradient echo \\
Imaging plane (orientation) & Transverse \\
Central slice or volume center & Centered on: \\
& Cervical spine: thyroid cartilage \\
& Thoracic spine: nipple line \\
Echo time $\left(T_{\mathrm{E}}\right)$ & Lumbar spine: iliac crests \\
Repeat time $\left(T_{\mathrm{R}}\right)$ & As short as possible \\
Flip angle $(\mathrm{FA})$ & As short as possible \\
Fields of view $\left(\mathrm{FOV}, \mathrm{F}_{\mathrm{x}}\right)$ & $15^{\circ}$ \\
& Cervical: $240 \mathrm{~mm}, 240 \mathrm{~mm}$ \\
& Thoracic: $320 \mathrm{~mm}, 320 \mathrm{~mm}$ \\
Resolution $(\Delta x, \Delta y)$ & Lumbosacral: $280 \mathrm{~mm}, 280 \mathrm{~mm}$ \\
& Cervical: $0.94 \mathrm{~mm}, 0.94 \mathrm{~mm}$ \\
Number of data points collected $\left(N_{\mathrm{x}}, N_{\mathrm{y}}\right)$ & Thoracic: $1.25 \mathrm{~mm}, 1.25 \mathrm{~mm}$ \\
Display matrix $\left(D_{\mathrm{x}}, D_{\mathrm{y}}\right)$ & Lumbosacral: $1.09 \mathrm{~mm}, 1.09 \mathrm{~mm}$ \\
Slice thickness $(\Delta z)$ & 256,256 \\
Number of slices & 5 mm \\
Slice gap & Variable \\
Number of acquisitions $\left(N_{\mathrm{acq}}\right)$ & Not applicable \\
Scan time & 1 \\
& $\sim 10$ sec \\
&
\end{tabular}

of special emergency equipment during the scanning procedure, or necessitate any other precautions.

Generally standard screening forms are used for all patients scanned in a magnetic resonance system.

The presence of any ferromagnetic metals may be a health hazard to the patient when he or she is inside the magnet, and will also affect the imaging. If in doubt as to the exact composition of the items, it is best to exclude patients with any metal implants; see Shellock (1996) for discussion of what implants may be safely scanned using magnetic resonance.

Patients may be accompanied into the magnet room by a friend or family member, who can sit in the room during the scan and comfort the patient as needed. This companion must be screened as well to ensure the absence of loose metal objects on the body or clothing.

2. If the procedure is a research protocol, have the patient sign any necessary consent form.

3. Have the patient remove all jewelry and change into a gown to eliminate any metal that might be found in clothing.

4. Inform the patient about what will occur during the procedure, what he or she will experience while in the magnet, and how to behave, including the following.

a. If earphones or headphones are used to protect the ears from the loud sounds produced by the gradients, the patient will be asked to wear these, but will be able to communicate with you at any time during the imaging.

b. The patient will be given a safety squeeze-bulb or similar equipment to request assistance at any time (demonstrate how this works). 
Table A8.5.3 Primary Clinical Imaging Parameters for Sequence 2 ( $T_{1}$-Weighted Image)

\begin{tabular}{|c|c|}
\hline Patient position & Supine \\
\hline Scan type & Conventional spin echo \\
\hline Imaging plane (orientation) & Sagittal \\
\hline \multirow[t]{4}{*}{ Central slice or volume center } & Centered on: \\
\hline & Cervical: 3rd cervical vertebra \\
\hline & Thoracic: 6 th thoracic vertebra \\
\hline & Lumbar: 3rd lumbar vertebra \\
\hline Echo time $\left(T_{\mathrm{E}}\right)$ & $10 \mathrm{msec}$ \\
\hline Repeat time $\left(T_{\mathrm{R}}\right)$ & $500 \mathrm{msec}$ \\
\hline Flip angle (FA) & $90^{\circ}$ \\
\hline \multirow[t]{3}{*}{ Fields of view $\left(\mathrm{FOV}_{\mathrm{x}}, \mathrm{FOV}_{\mathrm{y}}\right)$} & Cervical: $240 \mathrm{~mm}, 240 \mathrm{~mm}$ \\
\hline & Thoracic: $320 \mathrm{~mm}, 320 \mathrm{~mm}$ \\
\hline & $\begin{array}{l}\text { Lumbosacral: } 280 \mathrm{~mm}, 280 \mathrm{~mm} \text { (may } \\
\text { use rectangular field of view, e.g., half } \\
\text { or three-quarter field, if available, or } \\
\text { tailor to region of interest) }\end{array}$ \\
\hline \multirow[t]{3}{*}{ Resolution $(\Delta x, \Delta y)$} & Cervical: $0.94 \mathrm{~mm}, 0.94 \mathrm{~mm}$ \\
\hline & Thoracic: $1.25 \mathrm{~mm}, 1.25 \mathrm{~mm}$ \\
\hline & Lumbosacral: $1.09 \mathrm{~mm}, 1.09 \mathrm{~mm}$ \\
\hline Number of data points collected $\left(N_{\mathrm{x}}, N_{\mathrm{y}}\right)$ & 256,256 \\
\hline Display matrix $\left(D_{\mathrm{x}}, D_{\mathrm{y}}\right)$ & 256,256 \\
\hline \multirow[t]{3}{*}{ Slice thickness $(\Delta z)$} & Cervical: $3 \mathrm{~mm}$ \\
\hline & Thoracic: $3 \mathrm{~mm}$ \\
\hline & Lumbar: 4 mm \\
\hline Number of slices & $\begin{array}{l}10 \text {, or more, as needed to cover the } \\
\text { region of interest }\end{array}$ \\
\hline \multirow[t]{3}{*}{ Slice gap } & Cervical: $0.5 \mathrm{~mm}$ \\
\hline & Thoracic: $1 \mathrm{~mm}$ \\
\hline & Lumbar: $1 \mathrm{~mm}$ \\
\hline Number of acquisitions $\left(N_{\mathrm{acq}}\right)$ & 2 \\
\hline Flow compensation & Yes (if available) \\
\hline Saturation pulses & $\begin{array}{l}\text { Yes; anterior cervical/thoracic/lumbar } \\
\text { slab to saturate larynx/vessels }\end{array}$ \\
\hline Slice series & $\begin{array}{l}\text { Left to right or the reverse depending } \\
\text { on preference }\end{array}$ \\
\hline Scan time & $\sim 4 \min$ \\
\hline
\end{tabular}

c. For good results the patient should not talk, and should avoid or minimize swallowing or other movement, during each scan-i.e., as long as the banging sounds continue. Between scans, talking and swallowing are allowed in most cases, but should be avoided when comparative positional studies are being performed; the patient will be informed when this is the case.

d. Nevertheless, the patient may call out at any time if he or she feels it necessary.

5. Have the patient mount onto the table. Either before or right after the patient lies down, set up any triggering devices or other monitoring equipment that is to be used.

6. Center the coil over the region where the key information is desired.

Make sure that the body is constrained to prevent motion, especially if high-resolution scans are to be run.

Extradural Spine

A8.5.3

Supplement 2 
Table A8.5.4 Primary Clinical Imaging Parameters for Sequence $3\left(T_{2}\right.$-Weighted Image, FSE) $^{a}$

Patient position

Scan type

Imaging plane (orientation)

Central slice or volume center

Echo time $\left(T_{\mathrm{E}}\right)$

Echo train length (ETL)

Repeat time $\left(T_{\mathrm{R}}\right)$

Flip angle (FA)

Fields of view $\left(\mathrm{FOV}_{\mathrm{x}}, \mathrm{FOV}_{\mathrm{y}}\right)$

Resolution $(\Delta x, \Delta y)$

Number of data points collected $\left(N_{\mathrm{x}}, N_{\mathrm{y}}\right)$

Display matrix $\left(D_{\mathrm{x}}, D_{\mathrm{y}}\right)$

Slice thickness $(\Delta z)$

Number of slices

Slice gap

Number of acquisitions $\left(N_{\text {acq }}\right)$

Flow compensation

Saturation pulses

Fat suppression

Slice series
Supine

Fast spin echo

Sagittal

Centered on area of interest (as in sequence 2, Table A8.5.3)

$100 \mathrm{msec}$

8

$4000 \mathrm{msec}$

$90^{\circ}$

As in sequence 2, Table A8.5.3

Cervical: $0.47 \mathrm{~mm}, 0.47 \mathrm{~mm}$

Thoracic: $0.63 \mathrm{~mm}, 0.63 \mathrm{~mm}$

Lumbosacral: $0.55 \mathrm{~mm}, 0.55 \mathrm{~mm}$

512,512

512, 512

Cervical: $3 \mathrm{~mm}$

Thoracic: $3 \mathrm{~mm}$

Lumbar: $4 \mathrm{~mm}$

Varies with spinal level

Cervical: $0.5 \mathrm{~mm}$

Thoracic: $1 \mathrm{~mm}$

Lumbar: $1 \mathrm{~mm}$

1

Yes (if available)

Yes; anterior cervical/thoracic/lumbar slabs to saturate larynx/vessels/heart

Yes

Left to right or the reverse depending on preference

$\sim 4 \min$

Scan time

${ }^{a}$ FSE, fast spin echo.

7. If needed, place a pillow or other support under the knees to make the patient more comfortable.

8. Use the centering light to position the patient (cervical spine: thryoid cartilage; thoracic spine: nipple line; lumbar spine: iliac crests) and put him or her into the center of the magnet.

Once this step has been performed, so long as the patient does not move on the table, the table itself can be moved and then replaced in the same position as before without jeopardizing the positioning of one scan relative to another.

9. If the patient is unable to hold still, provide an appropriate sedative.

\section{Sequence 1: Rapid positioning pilot}

10. To validate the patient's position, run the system's pilot (or scout) scan (sequence 1) to ensure correct location of the neck in three dimensions, using the imaging sequence given in Table A8.5.2 or similar parameters.

Spinal Primary Neoplasia/ Metastasis

A8.5.4

This sequence usually consists of three orthogonal planes to allow subsequent localization. The images are often also used later to determine where to place the saturation pulses and to set up total coverage of the volume of interest. 
Table A8.5.5 Primary Clinical Imaging Parameters for Sequence 4 ( $T_{1}$-Weighted Image)

\begin{tabular}{|c|c|}
\hline Patient position & Supine \\
\hline Scan type & Conventional spin echo \\
\hline Imaging plane (orientation) & Transverse \\
\hline Central slice or volume center & $\begin{array}{l}\text { Centered on the area of interest (as in } \\
\text { sequence } 2 \text {, Table A8.5.3) }\end{array}$ \\
\hline Echo time $\left(T_{\mathrm{E}}\right)$ & $10 \mathrm{msec}$ \\
\hline Repeat time $\left(T_{\mathrm{R}}\right)$ & $500 \mathrm{msec}$ \\
\hline Flip angle (FA) & $90^{\circ}$ \\
\hline Fields of view $\left(\mathrm{FOV}_{\mathrm{x}}, \mathrm{FOV}_{\mathrm{y}}\right)$ & As in sequence 2 , Table A8.5.3 \\
\hline \multirow[t]{3}{*}{ Resolution $(\Delta x, \Delta y)$} & Cervical: $0.94 \mathrm{~mm}, 0.94 \mathrm{~mm}$ \\
\hline & Thoracic: $1.25 \mathrm{~mm}, 1.25 \mathrm{~mm}$ \\
\hline & Lumbosacral: $1.09 \mathrm{~mm}, 1.09 \mathrm{~mm}$ \\
\hline Number of data points collected $\left(N_{\mathrm{x}}, N_{\mathrm{y}}\right)$ & 256,256 \\
\hline Display matrix $\left(D_{\mathrm{x}}, D_{\mathrm{y}}\right)$ & 256,256 \\
\hline \multirow[t]{3}{*}{ Slice thickness $(\Delta z)$} & Cervical: $3 \mathrm{~mm}$ \\
\hline & Thoracic: $3-8 \mathrm{~mm}$ \\
\hline & Lumbar: 4 mm \\
\hline Number of slices & Varies with spinal level \\
\hline \multirow[t]{3}{*}{ Slice gap } & Cervical: $1 \mathrm{~mm}$ \\
\hline & Thoracic: $1-2 \mathrm{~mm}$ \\
\hline & Lumbar: $1 \mathrm{~mm}$ \\
\hline Number of acquisitions $\left(N_{\text {acq }}\right)$ & 2 \\
\hline Slice locations & See text (Basic Protocol, step 16) \\
\hline Saturation pulses & No \\
\hline Scan time & $\sim 4 \mathrm{~min}$ \\
\hline
\end{tabular}

\section{Sequence 2: Sagittal $T_{1}$-weighted conventional spin echo}

11. Set the imaging parameters as shown in Table A8.5.3.

12. Use the pilot image to locate the spine in three dimensions to ensure coverage of the region of interest (e.g., cervical, thoracic, lumbosacral spine).

13. Let the patient know you are ready and begin the scan.

\section{Sequence 3: Sagittal $T_{2}$-weighted fast spin echo, fat suppressed}

14. Review the pilot scans and ensure that the saturation pulse is correctly placed anterior to above the slab of interest.

15. Run sequence 3 according to Table A8.5.4.

\section{Sequence 4: Transverse $T_{1}$-weighted conventional spin echo}

16. Using the midline sagittal $T_{1}$-weighted image acquired in sequence 2 , set the transverse acquisition parameters as follows:

a. Cervical spine: stacked images from $\mathrm{C} 1$ through C7-T1.

b. Thoracic spine: stacked images through levels of interest.

c. Lumbosacral spine: 5 slices each, angled to the plane of the intervertebral disc at L3-4, L4-5, and L5-S1; one slice each, angled to the intervertebral disc at L1-2 and $\mathrm{L} 2-3$.

17. Supplement additional slices according to visible disease present or to clinical query. 
Table A8.5.6 Primary Clinical Imaging Parameters for Sequence 5 ( $T_{2}$-Weighted Image, FSE) ${ }^{a}$

\begin{tabular}{ll}
\hline Patient position & Supine \\
Scan type & Fast spin echo \\
Imaging plane (orientation) & Transverse \\
Central slice or volume center & Centered on the region of interest (as \\
& in sequence 2, Table A8.5.3) \\
Echo time $\left(T_{\mathrm{E}}\right)$ & $100 \mathrm{msec}$ \\
Echo train length $(\mathrm{ETL})$ & 8 \\
Repeat time $\left(T_{\mathrm{R}}\right)$ & $4000 \mathrm{msec}$ \\
Flip angle $(\mathrm{FA})$ & $90^{\circ}$ \\
Fields of view $\left(\mathrm{FOV}_{\mathrm{x}}, \mathrm{FOV}_{\mathrm{y}}\right)$ & As in sequence 2, Table A8.5.3 \\
Resolution $(\Delta x, \Delta y)$ & Cervical: $0.94 \mathrm{~mm}, 0.94 \mathrm{~mm}$ \\
& Thoracic: $1.25 \mathrm{~mm}, 1.25 \mathrm{~mm}$ \\
& Lumbosacral: $1.09 \mathrm{~mm}, 1.09 \mathrm{~mm}$ \\
Number of data points collected $\left(N_{\mathrm{x}}, N_{\mathrm{y}}\right)$ & 256,256 \\
Display matrix $\left(D_{\mathrm{x}}, D_{\mathrm{y}}\right)$ & 256,256 \\
Slice thickness $(\Delta z)$ & Cervical: $3 \mathrm{~mm}$ \\
& Thoracic: $3-8 \mathrm{~mm}$ \\
Number of slices & Lumbar: $4 \mathrm{~mm}$ \\
Slice gap & Varies with spinal level \\
& Cervical: $1 \mathrm{~mm}$ \\
Number of acquisitions $\left(N_{\mathrm{acq}}\right)$ & Thoracic: $1-2 \mathrm{~mm}$ \\
Slice locations & Lumbar: $1 \mathrm{~mm}$ \\
Saturation pulses & 2 \\
Scan time & See text (Basic Protocol, step 16) \\
\hline
\end{tabular}

${ }^{a} \mathrm{FSE}$, fast spin echo.

18. Run the sequence according to Table A8.5.5.

\section{Sequence 5: Transverse $T_{2}$-weighted fast spin echo}

19. Using the midline $T_{1}$-weighted image acquired in sequence 2 , repeat the setup as in Table A8.5.6.

20. Run sequence 5 according to Table A8.5.6.

\section{Sequences 6 and 7: Sagittal and transverse i.v. contrast enhanced $T_{1}$-weighted spin echo}

21. Remove patient from the magnet. Use the same equipment and perform equipment and patient setup as in steps 5 to 9 .

22. Establish an i.v. line from which the contrast agent can be injected, and attach this line securely to the patient so that movement into or out of the magnet will not pull at the patient's arm. Put the patient back into the MR machine. The contrast agent will be used to assess the extent of the neoplasm.

It is preferable to insert the line prior to imaging and to leave the patient in the magnet, with no intervening motion, between the scans run before contrast agent injection and those run after injection.

Spinal Primary Neoplasia/ Metastasis A8.5.6

\section{Run pilot scan}

23. Run a rapid three-plane positioning pilot scan (see sequence 1). 
Scan sequences

24. Leaving the patient in the magnet, inject the contrast agent, flush the i.v. line with 10 $\mathrm{ml}$ saline, and then immediately run sagittal (sequence 6) and transverse (sequence 7) $T_{1}$-weighted image sequences (see sequences 2 and 4 ).

A dose of $0.1 \mathrm{mmol} / \mathrm{kg}$ of contrast agent is usually given.

Fat suppression techniques may be very useful in order to suppress osseous vertebral marrow fat and perispinal soft tissue fat, thereby clearly distinguishing enhancement from normal fatty tissue.

\section{CORONAL, CONTRAST ENHANCED ACQUISITION}

In some instances (e.g., neurofibromatosis with multiple, bilateral neoplasms extending through the neural foramina), a coronal acquisition may be helpful to analyze the perispinal tissues for tumor extension either inward to or outward from the central spinal canal.

\section{Sequence 8: Contrast enhanced coronal $T_{1}$-weighted image}

Run the sequence according to sequence 8, Table A8.5.7. As stated above, fat suppression may be useful.

Table A8.5.7 Primary Clinical Imaging Parameters for Sequence 8 ( $T_{1}$-Weighted Image)

\begin{tabular}{ll}
\hline Patient position & Supine \\
Scan type & Conventional spin echo \\
Imaging plane (orientation) & Coronal \\
Central slice or volume center & $\begin{array}{l}\text { Centered on the area of interest (as in } \\
\text { sequence 2, Table A8.5.3) }\end{array}$ \\
Echo time $\left(T_{\mathrm{E}}\right)$ & $10 \mathrm{msec}$ \\
Repeat time $\left(T_{\mathrm{R}}\right)$ & $500 \mathrm{msec}$ \\
Flip angle $(\mathrm{FA})$ & $90^{\circ}$ \\
Fields of view $\left(\mathrm{FOV}_{\mathrm{x}}, \mathrm{FOV}_{\mathrm{y}}\right)$ & As in sequence 2, Table A8.5.3 \\
Resolution $(\Delta x, \Delta y)$ & Cervical: $0.94 \mathrm{~mm}, 0.94 \mathrm{~mm}$ \\
& Thoracic: $1.25 \mathrm{~mm}, 1.25 \mathrm{~mm}$ \\
& Lumbosacral: $1.09 \mathrm{~mm}, 1.09 \mathrm{~mm}$ \\
Number of data points collected $\left(N_{\mathrm{x}}, N_{\mathrm{y}}\right)$ & 256,256 \\
Display matrix $\left(D_{\mathrm{x}}, D_{\mathrm{y}}\right)$ & 256,256 \\
Slice thickness $(\Delta z)$ & $3-4 \mathrm{~mm}$ \\
Number of slices & Varies with spinal level \\
Slice gap & $1-2 \mathrm{~mm}$ \\
Number of acquisitions $\left(N_{\mathrm{acq}}\right)$ & 2 \\
Slice locations & From front to back of spinal column \\
Saturation pulses & No \\
Fat suppression & Yes: fat saturation or STIR (short tau \\
Scan time & inversion recovery) \\
\hline
\end{tabular}

${ }^{a} \mathrm{FSE}$, fast spin echo.

Extradural Spine

A8.5.7 


\section{COMMENTARY}

\section{Background Information}

Primary bony tumors affecting the spinal column (e.g., osteosarcoma, chondrosarcoma, Ewing's sarcoma, myeloma, chordoma, giant cell tumor, aneurysmal bone cyst, osteoblastoma, etc.) are not common, but may present with epidural extension and clinically relevant compression of the spinal cord, spinal nerves, and/or cauda equina. The primary bony component may produce a pathologic vertebral fracture. These changes will be evident on MRI without the use of an intravenous contrast agent. However, intravenous gadolinium (Gd) can be very helpful in delineating the extent and degree of neural tissue compression, which aids in preoperative decompression surgical planning.

Malignant perispinal neoplasms (e.g., soft tissue sarcomas, renal cell carcinoma) and metastatic nodal diseases (including lymphoma) in the perispinal regions may secondarily involve the spinal column and spinal canal. Bony destruction and neural compromise may produce pain and neurologic deficits. MR clearly demonstrates any extension of the neoplasm into the spinal canal and the resulting effects on the spinal cord and/or cauda equina.

Following the lung and liver, the skeletal system is the most common site of metastatic neoplastic disease, and in the skeletal system, the spine is the most common area affected.

Hematogenously disseminated bony spinal neoplastic metastases are reported in up to $10 \%$ of patients with malignancies. The frequency of neoplastic metastases to the spine is greatest in the thoracic region, and least frequent in the cervical area. The involvement of the spine is most often multisegmental. The vertebral body tends to be involved first, with secondary extension into the pedicles. However, hematogenously disseminated metastases may occur directly to any spinal structure, including the posterior bony neural arch. At least 5\% of patients eventually will reveal subsequent epidural extension of the metastatic disease. Neoplasms commonly encountered involving the vertebrae and adjacent epidural space of the spine include carcinomas (e.g., lung, breast, or prostate carcinoma), lymphoma, and myeloma. Epidural tumor may compress the intraspinal neural structures (e.g., spinal cord, nerve roots) and cause consonant neurological deficits. Extension of tumor directly from the vertebral body into the anterior epidural space of the spinal cord may be divided in the midline and deflected laterally by the midline septum complex, a fibrous structure attached to the posterior longitudinal ligament and the posterior surface of the vertebral body. It has been shown repeatedly that radionuclide bone scanning is less sensitive than MR for the detection of bony metastatic disease of the spine. On MR, the bony metastases are seen to replace hyperintense marrow on $T_{1}$-weighted imaging with hypointense tumor/peritumoral edema; marrow tumor/edema becomes hyperintense on $T_{2^{-}}$ weighted acquisitions, and can be especially well visualized on fat-suppressed studies (Mehta et al., 1995). After i.v. contrast administration, the degree of abnormal contrast enhancement of the involved vertebral marrow varies from patient to patient and from location to location in the same patient. Epidural tumor on MR tends to be generally isointense to muscle tissue on $T_{1}$-weighted images and isointense or hyperintense on $T_{2}$-weighted acquisitions. Enhancement of the epidural tumor component after i.v. gadolinium administration is typically moderate to intense and usually homogeneous (Fig. A8.5.1); this enhancement may also vary in degree and pattern from location to location. The spinal cord and nerve roots are not infrequently seen to be compressed by the metastatic epidural tumor mass.

\section{Critical Parameters and Troubleshooting}

Cerebrospinal fluid (CSF) flow, cardiac, laryngeal, body wall and other sources of motion can produce artifacts that can on occasion significantly degrade the images. Proper spatial (e.g., prevertebral) saturation pulses and sometimes flow compensation pulses and/or cardiac/respiratory gating can reduce these artifacts significantly. In many instances these artifacts may be difficult or impossible to easily overcome from patient to patient.

Another problem that may surface during the MRI evaluation of patients with primary or metastatic disease of the spinal column is pain severe enough to prevent patient cooperation for the duration of a sometimes holospine (e.g., cervical, thoracic, lumbar spine) evaluation. For this reason, patients should be on call from the hospital floor with an immediately preceding dose of pain medication, and the physician in charge should be reachable by phone or in person in order to supplement the pain medication as needed in the MRI suite. In addition, all

\section{Spinal Primary Neoplasia/ Metastasis}

A8.5.8 
A

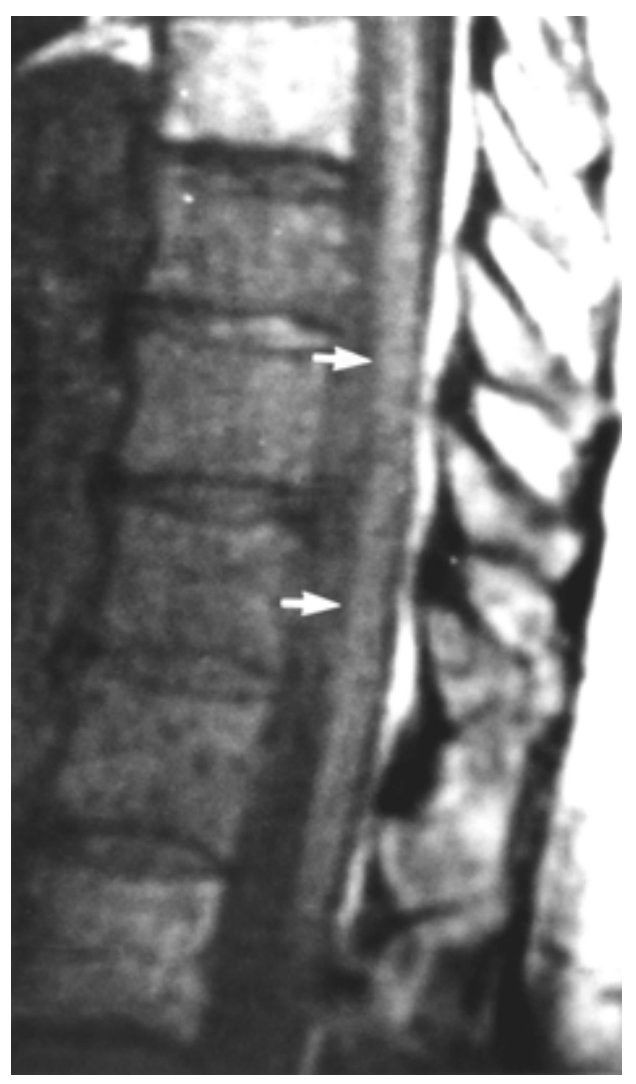

$\mathrm{B}$

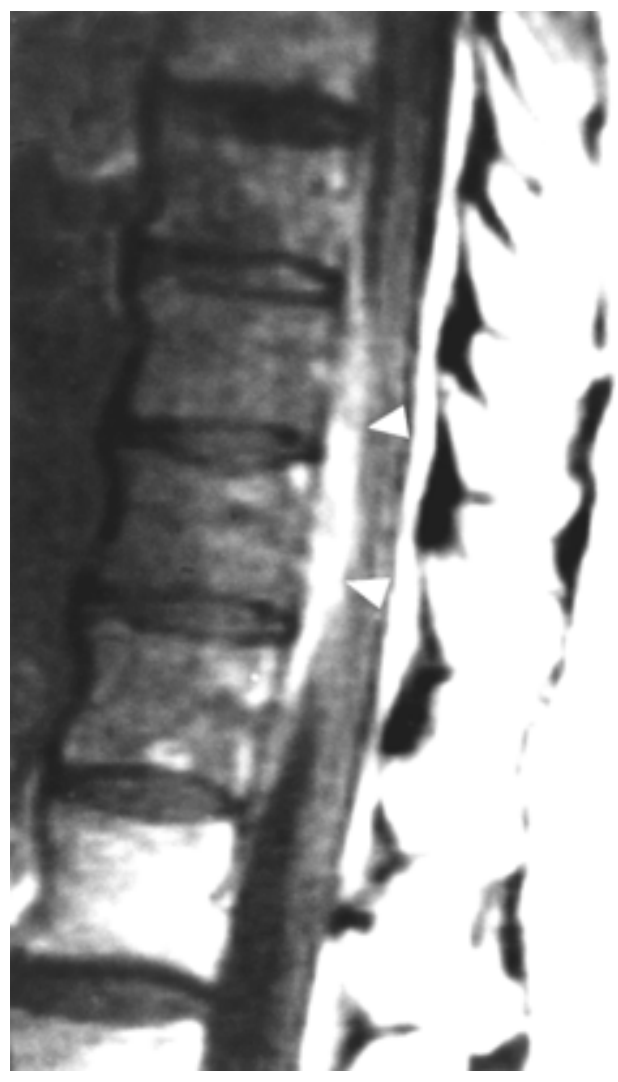

Figure A8.5.1 Spinal lymphoma with epidural extension. (A) Sagittal $T_{1}$-weighted ( $T_{\mathrm{R}}=500 \mathrm{msec}, T_{\mathrm{E}}$ $=10 \mathrm{msec}$ ) image shows hypointensity within the marrow of multiple thoracic vertebral bodies. Note that the spinal cord is displaced posteriorly (arrows). (B) Intravenous gadolinium enhanced $T_{1}$-weighted $\left(T_{\mathrm{R}}\right.$ $=500 \mathrm{msec}, T_{\mathrm{E}}=10 \mathrm{msec}$ ) image shows poor enhancement of the abnormal thoracic bodies and a multilevel enhancing epidural lymphomatous mass (arrowheads).

efforts should be undertaken by the technical staff to insure a speedy examination.

In some critical settings, simply $T_{1^{-}}$ weighted images in two planes (e.g., sagittal and transverse) before and after intravenous gadolinium administration may be all that is required to visualize clearly the extent of epidural tumor extension. Deleting the $T_{2^{-}}$ weighted acquisitions will speed up the overall examination time in cases when the entire spine must be examined in an emergency situation. This decision to curtail the examination must be made at the discretion of the imaging physician, taking each case into consideration individually.

\section{Anticipated Results}

The goal of studying the spine in cases of known or suspected neoplastic disease is to reveal the area or areas of involvement, the presence of secondary pathologic changes (e.g., pathologic fractures), and the degree and extent of epidural extension of tumor with neural tissue (e.g., spinal cord, cauda equina) compression. This information will allow the referring physician to accurately plan the type of therapy as well as the specific approach.

\section{Literature Cited}

Algra, P.R., Bloem, J.L., Tissing, H., Falke, T.H., Arndt, J.W., and Verboom, L.J. 1991. Detection of vertebral metastases: Comparison between MR imaging and bone scintigraphy. Radiographics 11:219-232.

Avrahami, E., Tadmor, R., Dally, O., and Hadar, H. 1989. Early MR demonstration of spinal metastases in patients with normal radiographs and CT and radionuclide bone scans. J. Comput. Assist. Tomogr. 13(4):598-602.

Beltran, J., Noto, A.M., Chakeres, D.W., and Christoforidis, A.J. 1987. Tumors of the osseous
Extradural Spine

\section{A8.5.9}

Supplement 2 
spine: Staging with MR imaging versus CT. $R a$ diology 162:565-569.

Carmody, R.F., Yang, P.J., Seeley, G.W., Seeger, J.F., Unger, E.C., and Johnson, J.E. 1989. Spinal cord compression due to metastatic disease: Diagnosis with MR imaging versus myelography. Radiology 173:225-229.

Coletti, P.M., Dang, H.T., Deseran, M.W., Kerr, R.M., Boswell, W.D., and Ralls, P.W. 1991. Spinal MR imaging in suspected metastases: Correlation with skeletal scintigraphy. Magn. Res. Imag. 9:335-349.

Colman, L.K., Porter, B.A., Redmond, J., III, Olson, D.O., Stimac, G.K., Dunning, D.M., and Friedl, K.E. 1988. Early diagnosis of spinal metastases by CT and MR studies. J. Comput. Assist. Tomogr. 12:423-426.

Daffner, R.H., Lupetin, A.R., Dash, N., Deeb, Z.L., Sefczek, R.J., and Schapiro, R.L. 1986. MRI in the detection of malignant infiltration of bone marrow. Am. J. Roentgenol. 146:353-358.

Jinkins, J.R. and Leite da Costa, C. 1998. Differential diagnosis of primary or secondary paraspinal abnormalities originating in the spine, perispinous tissues, or remote sources. In Neurodiagnostic Imaging Pattern Analysis and Differential Diagnosis (J. R. Jinkins and C. Da Costa Leite, ed.) pp. 773-779. Lippincott-Raven, Philadelphia.
Mehta, R.C., Marks, M.P., Hinks, R.S., Glover, G.H., and Enzmann, D.R. 1995. MR evaluation of vertebral metastases: $T_{1}$-weighted, short-inversion-time inversion recovery, fast spin-echo, and inversion-recovery fast spin-echo sequences. Am. J. Neuroradiol. 16:281-288.

Ragland, R.L., Knorr, J.R., Kamath, S.V., Landis, E.S., Tenreiro-Picon, O.R., and Weyreuther, M. 1996. Magnetic resonance patterns of epidural impression from spinal metastases: review of 200 cases. Int. J. Neuroradiol. 1:69-72.

Shellock, F.G. 1996. Pocket Guide to MR Procedures and Metallic Objects. Lippincott-Raven, Philadelphia.

Sugimura, K., Kajitani, A., Okizuka, H., Sugihara, M., Mizutani, M., and Ishida, T. 1991. Assessing response to therapy of spinal metastases with gadolinium-enhanced MR imaging. J. Magn. Reson. Imag. 1:481-484.

Sze, G., Krol, G., Zimmerman, R.D., and Deck, M.D. 1988. Malignant extradural spinal tumors: MR imaging with Gd-DTPA. Radiology 167:217-223.

Contributed by J. Randy Jinkins and David D. Stark

Downstate Medical Center

State University of New York

Brooklyn, New York 